\title{
A Study on the Application of Wavelet Packet Transform in Low-Strain Pile Integrity Test
}

\author{
[ Hyeong-Joo Kim, Jose Leo Mission, Tae-Woong Park, Peter Rey Dinoy, Hyeong-Soo Kim ]
}

\begin{abstract}
Nonlinear amplification of velocity signals in lowstrain integrity tests is typically done in order to enhance weak echoes and to superimpose any peak reflections. However, unraveling hidden information within signals obscured by noise may sometimes fail when using the conventional method. Therefore, a pile defect identification system is presented in this study based on nonlinear amplification and wavelet packet transform (WPT). After the conventional nonlinear amplification method is applied, the signals are decomposed by WPT in which the detection of the feature parameters from the presence of any geometric or material defects may easily be identified. To serve as a guide in the interpretation of the various characteristics of peak wave forms that are associated with defects, diagnostic rules are presented in this study. In addition, a parametric study was performed using the finite element method and results have shown that the identification method based on WPT can be a useful tool in identifying major defects within the effective length of the pile compared to signal amplification alone.
\end{abstract}

Keywords-diagnostic rule, pile defects, wavelet packet transform, nonlinear amplification method

\section{Introduction}

Difficult soils and hydrogeological conditions can cause pile defects and impair its constructed pile geometry and material quality, which reduces pile strength and capacity. Therefore, low strain testing such as the impact echo test method have been extensively used to evaluate pile integrity, uniformity, and continuity. Various guidelines are presented in literature regarding the interpretation of low strain integrity test results. In most cases, the typical method for signal processing and analysis of results in impact echo tests is by linear/nonlinear amplification of the original signal scale response to superimpose any reflected peaks and to enhance weak echoes. The basic method of scale amplification of the original signal alone may sometimes fail to identify local and hidden discontinuities, irregularities, and small defects for complex signals, whose interpretation are sometimes obscured by noise.

Hyeong-Joo Kim, Tae-Woong Park, Peter Rey Dinoy, Hyeong Soo-Kim

Kunsan National University

Gunsan, South Korea

Jose Leo Mission

Horizon Survey Company

SAIF Zone, Sharjah, United Arab Emirates
In this study, the wavelet packet transform (WPT) is applied for analyzing and clarifying the reflection signals in impact echo test of piles by decomposing their scale and time aspects into high resolution components to be able to reveal and identify any hidden information due to the presence of defects, flaws, anomalies, imperfections, material inconsistencies, or damage.

\section{Low- Strain Pile Integrity Test by Impact Echo Method}

The low-strain pile integrity test by impact echo method, as shown in Fig. 1, involves impacting the top of a deep foundation with a hammer to generate a downward travelling compression wave, which reflects back to the surface from changes in stiffness, cross-sectional area, and density. The arrival of the reflected wave energy is sensed by a receiver (accelerometer or vertical geophone). If defect is present along the shaft, its size and location can be estimated by analyzing the propagation and reflection of the wave signal induced on the foundation by the hammer impact. In addition, the depth of the pile toe can be estimated by impact echo method.

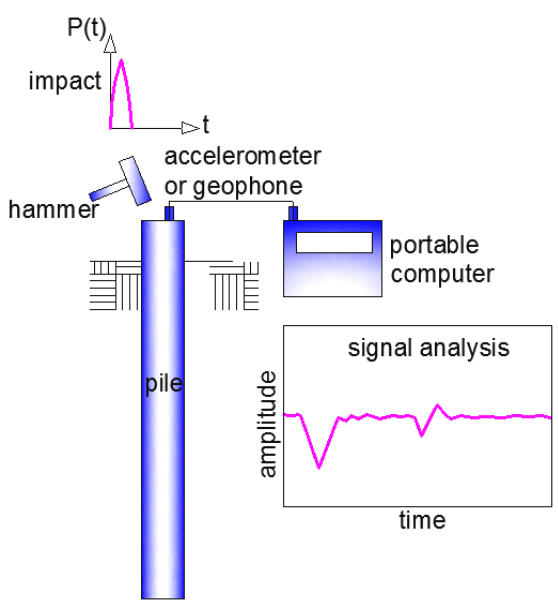

Figure 1. Low-strain pile integrity test by impact echo method.

At low strain level, the mobilized shaft resistance is small and may be neglected for all practical purposes, in which the low strain condition is formed as the basis for the integrity testing [1]. Considering the pile to be a one-dimensional elastic and isotropic bar, the equation of motion of the stress wave propagating along the axis in a pile-soil system is given by Eqs. (1) and (2) [2].

$$
\partial p / \partial t+\zeta \partial v / \partial x=0
$$




$$
\partial p / \partial x+\zeta \partial v / \partial t=0
$$

where $p(x, t)$ and $v(x, t)$ denote the force and particle velocity acting on each pile section, respectively, $\zeta$ is defined as the wave impedance of the pile, $t$ is the time measured from the moment of impact, and $x$ is the travel time. For a perfectly intact and uniform pile with length $L$ having no defect or imperfections, the reflected wave from the pile tip propagates a total distance of $2 L$. Therefore, the total travel time $t$ is given as,

$$
t=2 L / c .
$$

where $c$ is the wave speed in the pile. The impedance $\zeta$ is the unique model parameter we seek to recover or interpret, with the wave Eqs. (1) and (3) as an inverse medium problem. The change in wave impedance at the defect is mainly due to the change in cross section area or wave velocity. The changes in impedance can be used to detect potential defects, anomalies, and imperfections such as major cracks, necking, soil inclusions or voids, low quality concrete, changes in pile cross section. Reflections from the pile tips can be used to determine the embedment length.

For the case of cast-in-place concrete drilled piles or bored shafts, the embedment depth or length $L$ is normally known with sufficient accuracy, such that the propagation time $t$ in Eq. (3) is mainly dependent and inversely proportional to the wave velocity of concrete $(c)$. The wave velocity is also regarded as an indication of concrete quality since the modulus of elasticity $E$ is related to the compressive strength $\left(f^{\prime} c\right)$ as shown in Table 1. In this study, the characteristics of the reflection time and impedance of the wave signals are explored as they relate to the presence of various geometric and material defects, imperfections, flaws, and anomalies in a pile.

TABLE I. RELATIONSHIP BETWEEN CONCRETE QUALITY, WAVE SPEED, COMPRESSIVE STRENGTH, AND MODULUS OF ELASTICITY

\begin{tabular}{|c|c|c|c|}
\hline $\begin{array}{c}\text { Concrete } \\
\text { Quality }\end{array}$ & $\begin{array}{c}\text { Impact Wave Speed } \\
\text { in Concrete, } \boldsymbol{c} \text { (m/s) }\end{array}$ & $\begin{array}{c}\text { Compressive } \\
\text { strength, } \boldsymbol{f} \mathbf{c} \\
\text { (MPa) }\end{array}$ & $\begin{array}{c}\text { Modulus of } \\
\text { Elasticity, } \boldsymbol{E} \\
\text { (MPa) }\end{array}$ \\
\hline Excellent & $>4570$ & $>73.5$ & $>40,294$ \\
\hline Good & $3660-4570$ & $20.4-73.5$ & $21,228-40,294$ \\
\hline Not good & $3050-3660$ & $8.6-20.4$ & $13,783-21,228$ \\
\hline bad & $2130-3050$ & $2.4-8.6$ & $7,281-13,783$ \\
\hline Very bad & $<2130$ & $<2.4$ & $<7,281$ \\
\hline
\end{tabular}

\section{Principle of Wavelet Packet Transf orm}

The principle of wavelet packet transform (WPT) method can be described as a generalization of the wavelet decomposition that offers a richer range of possibilities for signal analysis that can be used in data preprocessing for fault diagnosis [3], damage identification [4], improving the time resolution and noise suppression [5], and signal compression [6]. In wavelet packet analysis, a signal is split into an approximation and a detail, which represent the low and high frequencies, respectively. The approximation is then itself split into a second-level approximation and detail, and the process is repeated. This decomposition in effect halves the time resolution, and doubles the frequency resolution. After WPT is successively applied, lower dimensional data with improved resolution can be obtained that would facilitate the defect identification and detection process.

\section{Identification and Classification of Defects}

An identification and classification scheme was designed to be able to distinguish the type of defect, if any, that would be present in the pile. Two main classifications are being made: first, based on comparisons of the measured pile tip reflection time $(T)$ with the reference time $(T r)$; and second, based on the number of peak reflections $(N)$.

A perfectly intact and uniform pile with no geometric and material defect would have a total reflection time $(T)$ almost equal to the reference time ( $T r$ ) for normal quality condition of the concrete material, and the number of reflection peaks is just two. When the measured tip reflection time $T<T r$, it signifies that the quality of the concrete material is higher than expected, and when $T>T r$ signifies the presence of any low quality material defect.

The presence of intermediate peaks greater than or equal to two signifies a geometric or material defect. Geometric defects such as the presence of neck, notch, crack, or voids are all generally categorized as a reduction in area of the pile crosssection, while bulge defects are generally categorized as enlargement in area. Based on comparisons of the directions or signs of the peak of the WPT processed signals, pile defects due to reduction in area or having material defects have a downward direction or negative sign of the first reflected wave and then an upward or positive sign of the next reflected wave, thus having the sign $(-/+)$, as shown in Fig. 2. On the other hand, the presence of geometric pile defect with enlarged section have an upward direction or positive sign of the first reflected wave and then a downward or negative sign of the next reflected wave, thus having the sign (+/-), as shown in Fig. 3.

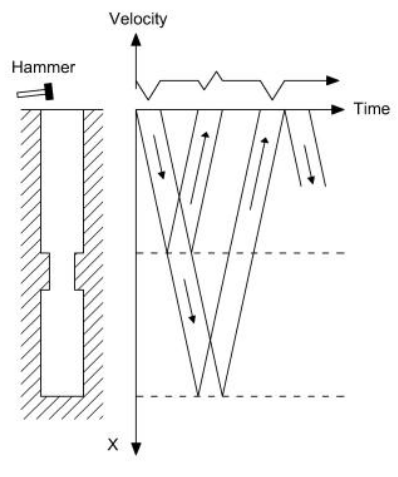

Figure 2. Pile with neck defect. 


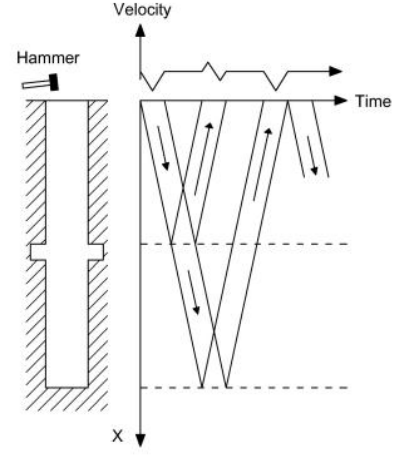

Figure 3. Pile with bulge imperfection.

All these observations can be clearly manifested and identified after WPT decomposition is applied. The proposed algorithm for the pile defect identification scheme is graphically presented in the flowchart diagrams shown in Figs. 4(a) and 4(b).
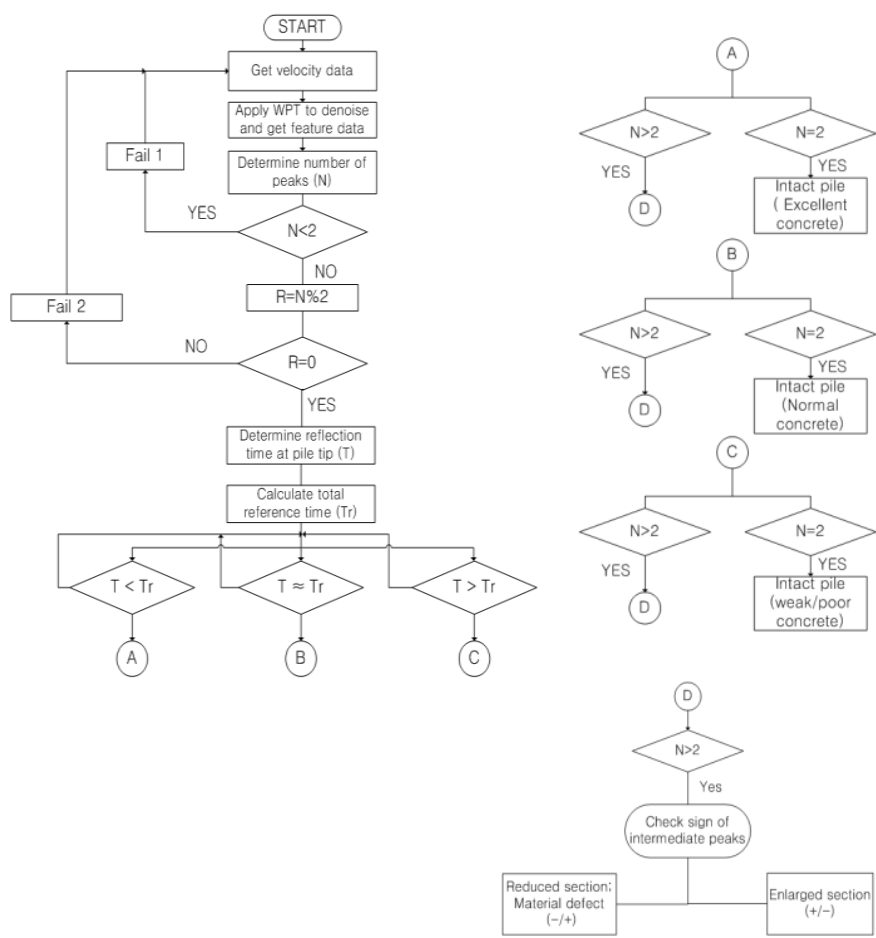

(a)

(b)

Figure 4. (a) Flowchart for pile defect diagnosis and (b) Detailed flowchart for diagnosis based on reference reflection time and number of peaks.

\section{v. Verification of Proposed Scheme by Parametric Study}

In order to test and verify the performance of the proposed scheme based on wavelet packet transform (Method 1), a parametric study was performed on defective piles using the finite element method by varying the various parameters and conditions of the simulated pile cases shown in Figs. 5(b-j) into 6 more subcases by varying the input parameters such as modulus of elasticity, and extent and location of the defects. The objective of the parametric study is to compare the conventional method (Method 2) with Method 1. To assess the performance of the two methods, detection ratios rated as high $=1.0$, medium $=0.5$, and low $=0.0$ were used.

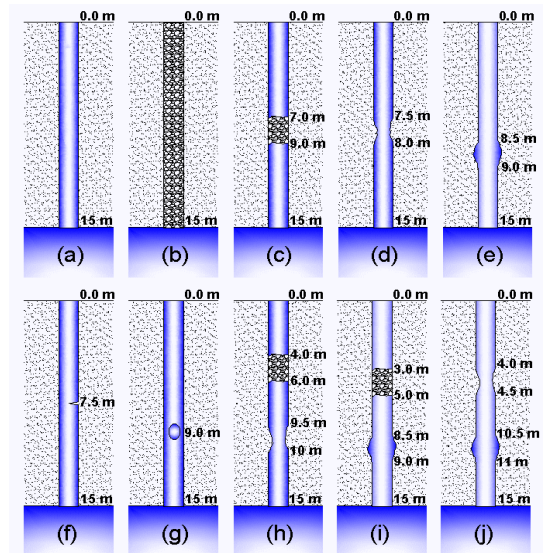

Figure 5. Parametric study: (a) intact and uniform pile, (b) pile with weak concrete throughout the length, (c) weak concrete at a section, (d) neck defect, (e) bulge imperfection, (f) notch, crack, or break, (g) void, and (h-j) multiple defects.

Comparisons between Method 1 and Method 2 are shown in Figs. 6-10. Results clearly show the identified feature parameters and noise suppression of the signal using Method 1. However, based on the observations of the velocity waveforms for pile defects due to reduction in area of the pile section, it is not possible to distinguish among a neck, crack, and void when the length of the neck or void is short, as shown in Figs. 7 and 10. Similarly, a very short extent of a weak pile segment might be misinterpreted to be a defect due to reduction in cross-section area, as shown in Fig. 6, especially when the measured reflection time at the pile tip $T$ is almost equal to the reference time $T_{r}$. The reflections from a reduced pile area defect can also be distinguished from a notch or crack if the extent of the defect is long, as shown in Fig. 9, although it may be difficult to distinguish it from being a long necking or a long and narrow void.
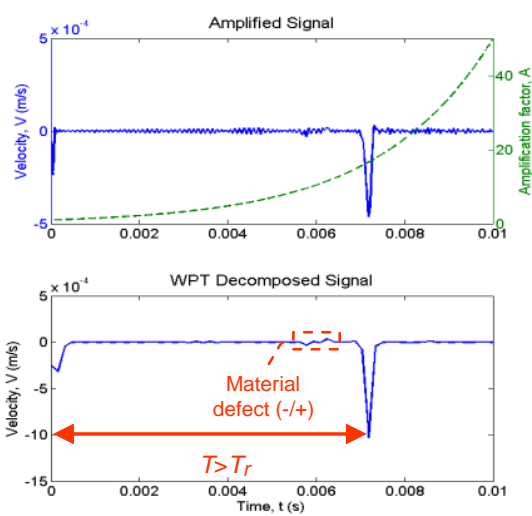

Figure 6. Amplified and WPT decomposed velocity signal for case C (pile with low quality concrete segment). 

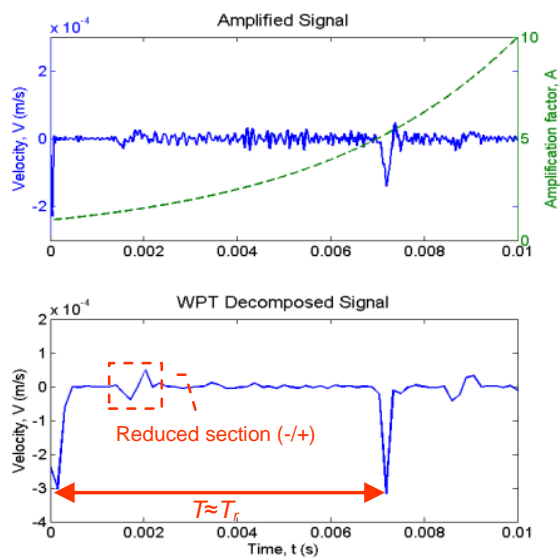

Figure 7. Amplified and WPT decomposed velocity signal for case D (neck defect).
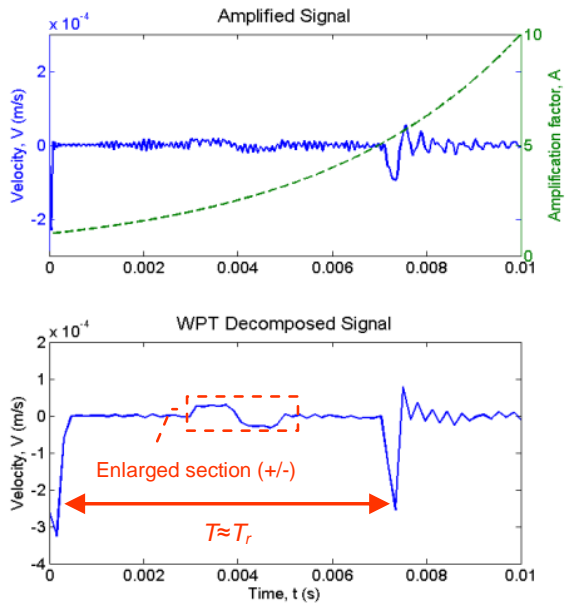

Figure 8. Amplified and WPT decomposed velocity signal for case E (bulge imperfection).
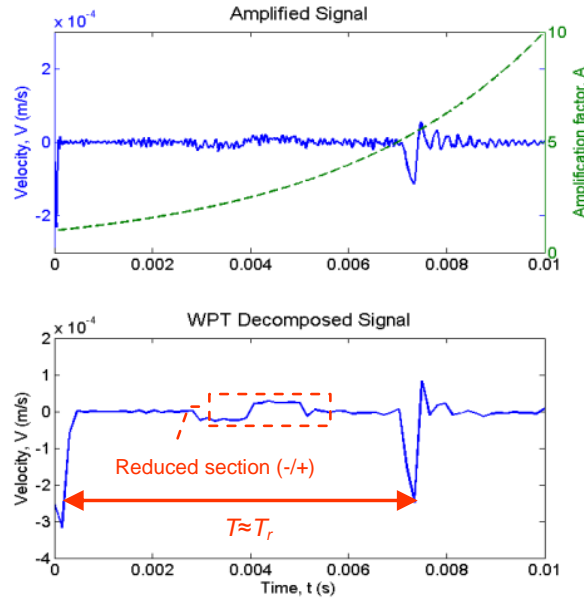

Figure 9. Amplified and WPT decomposed velocity signal for Case F (notch, crack, or break).
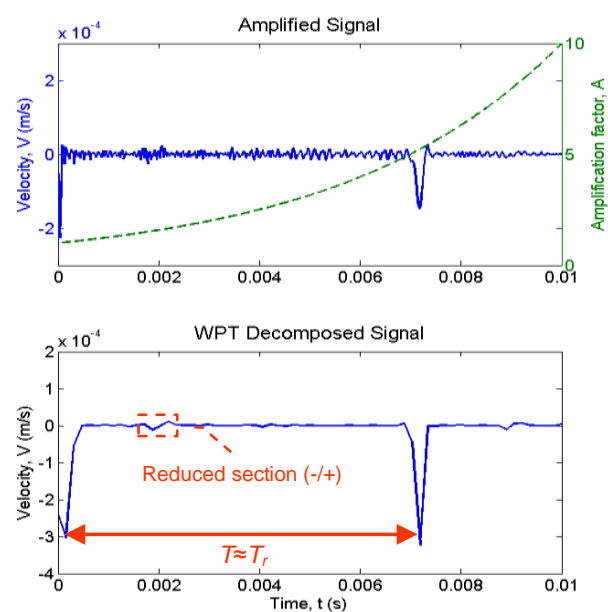

Figure 10. Amplified and WPT decomposed velocity signal for Case G (void).

There are also certain magnitudes of defect in which identification by WPT is low or not possible such as when the extent of the neck, bulge, depth of notch or crack, and maximum size of void is smaller than about $10 \%$ of the pile diameter. These phenomena of limitations in pile integrity testing are also presented by Tchepak [7] and Hartung et al. [8]. Nevertheless, the identification method based on WPT coupled with the conventional amplification method can be a useful tool in identifying major defects within the effective length of the pile compared to the method by amplification alone as verified by the comparison of the detection performance rating shown in Table 2 .

TABLE II. COMPARISON OF PERFORMANCE RATING OF PILE DEFECT DIAGNOSIS FROM PARAMETRIC STUDY

\begin{tabular}{|c|c|c|}
\hline Case & $\begin{array}{c}\text { Method 1 (Nonlinear } \\
\text { amplification and } \\
\text { WPT) }\end{array}$ & $\begin{array}{c}\text { Method 2 (Nonlinear } \\
\text { amplification) }\end{array}$ \\
\hline B & $100 \%$ & $100 \%$ \\
\hline C & $86 \%$ & $57 \%$ \\
\hline D & $93 \%$ & $50 \%$ \\
\hline E & $93 \%$ & $50 \%$ \\
\hline F & $71 \%$ & $43 \%$ \\
\hline G & $86 \%$ & $64 \%$ \\
\hline H & $93 \%$ & $86 \%$ \\
\hline I & $86 \%$ & $79 \%$ \\
\hline J & $93 \%$ & $86 \%$ \\
\hline Total & $89 \%$ & $68 \%$ \\
\hline
\end{tabular}

\section{vi. Conclusions}

In this study, the application of the wavelet packet transform (WPT) was demonstrated for the efficient noise suppression, identification of the feature parameters, and detection of defects in the integrity testing of piles by impact echo test method. Diagnostic rules were presented to provide a guideline for a more efficient interpretation of the identified feature parameters in an impact echo test. A parametric study 
was performed on defective piles using the finite element method by varying the various parameters and conditions of the simulated pile cases such as modulus of elasticity, and extent and location of the defects. Results clearly show the identified feature parameters and noise suppression of the signal using WPT. However, based on the observations of the velocity waveforms for pile defects due to reduction in area of the pile section, it is not possible to distinguish among a neck, crack, and void when the length of the neck or void is short. Similarly, a very short extent of a weak pile segment might be misinterpreted to be a defect due to reduction in cross-section area, especially when the measured reflection time at the pile tip $(T)$ is almost equal to the reference time $\left(T_{r}\right)$. The reflections from a reduced pile area defect can also be distinguished from a notch or crack if the extent of the defect is long, although it may be difficult to distinguish it from being a long necking or a long and narrow void. Even though the integrity testing on piles may not identify all minor imperfections, the results of the study have shown that the identification method based on WPT coupled with the conventional amplification method can be a useful tool in identifying major defects within the effective length of the pile compared to the method by signal amplification alone.

\section{Acknowledgment}

This research was supported by the Basic Science Research Program through the National Research Foundation of Korea (NRF) funded by the Ministry of Education (NRF591 2017R1D1A3B03034189).

\section{References}

[1] T. Horiguchi, M. Karkee, T. Sakai, and H. Kishida, "Assessment of the pile integrity and the mobilized bearing resistance using the mid-range dynamic test," Proceedings of the Fifth International Conference on the Application of Stress-Wave Theory to Piles (StressWave '96), Orlando Florida, September 1996, pp. 971-979.

[2] J. Wang and M. Ding, "Quantitative analysis of pile integrity," Proceedings of the Fifth International Conference on the Application of Stress-Wave Theory to Piles (StressWave '96), Orlando, Florida, September 1996, pp. 939-944.

[3] B. Liu, "Selection of wavelet packet basis for rotating machinery fault diagnosis," J. of Sound and Vibration, vol. 284, pp. 567-582, 2005.

[4] J.G. Han, W.X. Ren, and Z.S. Sun, "Wavelet packet based damage identification of beam structures." Int. J. of Solids and Struct., vol. 42, pp. 6610-6627, 2005.

[5] F. Bettayeb, S. Haciane, and S. Aoudia, "Improving the time resolution and signal noise ratio of ultrasonic testing of welds by the wavelet packet," Nondestructive Testing and Evaluation (NDT\&E) Int., vol. 38, pp. 478-484, 2005.

[6] B. Walczak and D.L. Massart, "Tutorial: Noise suppression and signal compression using the wavelet packet transform," Chemometrics and Intelligent Laboratory Systems, vol. 36, pp. 81-94, 1997.

[7] S. Tchepak, "Experiences with low strain integrity testing of cast in situ piles," Proceedings of the Fifth International Conference on the Application of Stress-Wave Theory to Piles (StressWave '96), Orlando, Florida, September 1996, pp. 612-625.

[8] M. Hartung, K. Meier, and W. Rodatz, "Integrity testing of model piles," Proceedings of the Fourth International Conference on the Application of Stress-Wave Theory to Piles, The Netherlands, 1992, pp. 265-269. 